\title{
Global Existence of Time-Dependent Yang-Mills-Higgs Monopoles
}

\author{
Jürgen Burzlaff ${ }^{1}$ and Niall O'Murchadha ${ }^{2}$ \\ 1 School of Theoretical Physics, Dublin Institute for Advanced Studies, 10 Burlington Road, \\ Dublin 4, Ireland \\ 2 Physics Department, University College, Cork, Ireland
}

\begin{abstract}
We study the Cauchy problem for non-abelian Yang-Mills-Higgs theory in (3+1)-dimensional Minkowski spacetime. With suitable conditions on the background fields and a suitable choice of a Sobolev space for the subtracted gauge potentials and the Higgs field, we establish local existence. We then prove global existence by showing that an appropriate norm of the solutions cannot blow up in a finite time.
\end{abstract}

\section{Introduction}

Irving Segal [1], in 1963, introduced a general existence theory for semi-linear evolution equations. In 1979 Segal [2] himself showed that classical Yang-Mills theory could be cast into a suitable form to make use of this general theory and he showed that the local-in-time Cauchy problem could be solved. This means that if one is given regular initial data on $\mathbb{R}^{3}$ at some initial time (call it $t=0$ ), there exists a unique smooth solution to the field equations compatible with the initial data over some finite time interval $\left(-t_{0}, t_{0}\right)$.

This result was improved on in 1982 by Ginibre and Velo [3] who added a Higgs field to the Yang-Mills potential and showed that local-in-time existence still held. The next major step forward was achieved, also in 1982, by Eardley and Moncrief [4] who independently derived the local existence result for Yang-MillsHiggs theory and then extended this to obtain a global existence proof, that is to show that the time existence interval $\left(-t_{0}, t_{0}\right)$ can be made unboundedly large.

The major interest in classical solutions to the Yang-Mills-Higgs equations is due to the existence of magnetic monopole solutions (which demand that the Yang-Mills potential A falls off like 1/r) and non-trivial topologies in the Higgs field (which require that the Higgs field remain finite at infinity). However, all the existence results to date demand that both the Higgs field and the Yang-Mills potential be square-integrable on $\mathbb{R}^{3}$, which naively demands that everything fall off faster than $r^{-3 / 2}$, and are incompatible with the magnetic monopoles and with non-trivial topologies. 
In this paper we will give a global existence proof for a class of Yang-MillsHiggs solutions which include solutions with magnetic monopoles and non-trivial topologies. The result we obtain here is not only interesting for its own sake; it is also immediately useful. For example, there has been some interest recently in approximation methods to study monopole-monopole scattering (e.g. Manton [5] and Atiyah and Hitchin [6]). In any approximation method one had better be sure that the approximate solution is an approximation to some exact solution, otherwise one may get nonsense (e.g. linearization instabilities in general relativity). The proof we give here should act as an underpinning to all these approximation techniques.

The method used in this paper to prove the desired existence theorem is quite straightforward. We specify, as part of the initial data a static background YangMills potential $\AA$ and Higgs field $\stackrel{\circ}{\Phi}$. These are chosen so that their asymptotic behaviour permits a finite magnetic charge and non-trivial topology. We then write the total potential $\mathbf{A}$ as $\AA+\mathbf{a}$ and $\Phi$ as $\Phi^{+}+\varphi$ and regard a and $\varphi$ as the dynamical fields which are square integrable, and so fall off rapidly at infinity. We will describe this in detail in Sect. II.

Of the two local-in-time existence techniques the Eardley and Moncrief method is superior to the Ginibre and Velo method. This is due to the fact that Eardley and Moncrief have found a way to build the $\operatorname{div} \mathbf{E}=0$ constraint directly into the dynamical equations whereas Ginibre and Velo, following Segal, ignore it until the end, and then show that the dynamics are compatible with the constraint. This allows Eardley and Moncrief to prove existence with weaker conditions on the initial data than Ginibre and Velo require.

Unfortunately, the background subtraction method turns out to be incompatible with the Eardley-Moncrief local-in-time technique (which Eardley and Moncrief realised themselves.) Happily, the background subtraction method turns out to be entirely compatible with the Ginibre and Velo proof, and so our local existence proof is obtained by a straightforward extension of their technique. Of course, we have to include extra terms in our field equations which arise from the background static $\AA$, $\Phi$ fields, but these are easy to handle. We prove the local-intime existence result in Sect. III.

To turn the local existence result into a global existence proof we switch back to Eardley and Moncrief and copy their technique. The difficulties in the local proof are not relevant to the global part and so we can follow them. Of course we still have to worry about the background fields. A further problem is that we have to make the global part of the proof agree with the local part. This means that we have to extend the global proof to one degree of differentiability higher than Eardley and Moncrief require. We manage to deal with both of these problems and get our global result in Sect. IV.

This should not come as any great surprise. For any theory, the only situations where one would have local but not global existence is where we would have either some local loss of differentiability through some form of singularity developing or where nothing bad would happen at any point, but some integral over the whole space would blow up. In view of the hyperbolic nature of the field equations, the singularity formation should be a strictly local phenomenon, and should not be influenced by asymptotic conditions. The problem of the norms blowing up can 
only be avoided by sensible choice of norm. For us, the natural norms are the energy and the higher derivative pseudo-energies, and so we are not surprised when they remain finite.

\section{Background and Dynamical Fields}

We will study classical Yang-Mills-Higgs theory on Minkowski space with a Yang-Mills potential $A_{\mu}(t, \mathbf{x})$ and a Higgs scalar $\Phi(t, \mathbf{x})$. The field corresponding to $A_{\mu}$ is

$$
F_{\mu \nu}=\partial_{\mu} A_{\nu}-\partial_{\nu} A_{\mu}+\left[A_{\mu}, A_{\nu}\right] .
$$

The Lagrangian we will use is

$$
\mathscr{L}=-\frac{1}{4}\left\langle F_{\mu v}, F^{\mu v}\right\rangle+\left\langle D_{\mu} \Phi, D^{\mu} \Phi\right\rangle-\left(|\Phi|^{2}-1\right)^{2},
$$

where $D$ is the covariant derivative with $A_{\mu}$ as connection. This is the natural Lagrangian to use to get a non-trivial topology in the Higgs field at infinity and a magnetic monopole, together with finite energy. This is achieved by having $\Phi$ nonconstant at infinity but in such a way that $|\Phi| \rightarrow 1$ at infinity. In addition we require $D_{\mu} \Phi=\partial_{\mu} \Phi+A_{\mu} \Phi$ to fall off rapidly at infinity. This can be achieved because while we naively expect $\partial_{\mu} \Phi$ to fall off like (at best) $1 / r$ at infinity, we can assume $A_{\mu} \sim 1 / r$ at infinity, and so $A_{\mu} \Phi$ also falls off like $1 / r$. The two $1 / r$ terms in $\partial_{\mu} \Phi$ and $A_{\mu} \Phi$ can cancel to give a faster fall-off to $D_{\mu} \Phi$. This is how the standard static monopole solutions behave.

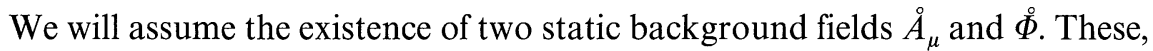
at least asymptotically, will behave like static monopole solutions. The actual potential and scalar fields we will write as

$$
\begin{gathered}
A_{\mu}(t, \mathbf{x})=\AA_{\mu}(\mathbf{x})+a_{\mu}(t, \mathbf{x}), \quad \AA_{0}=0, \\
\Phi(t, \mathbf{x})=\stackrel{\circ}{\Phi}(\mathbf{x})+\varphi(t, \mathbf{x}) .
\end{gathered}
$$

We will treat the subtracted fields $\left(a_{\mu}, \varphi\right)$ as the dynamical fields and demand that they fall off rapidly enough to give us finite energy solutions.

We will describe everything in terms of the Sobolev spaces $H_{k}$ with norm

$$
\|u\|_{H_{k}}^{2}=\sum_{|\alpha| \leqq k}\left\|\left|\partial^{\alpha} u\right|\right\|_{L_{2}}^{2}<\infty
$$

for various values of $k$, except $\AA$ and $\Phi$. We will assume that $\AA$ and $\Phi$ satisfy the following conditions

$$
\begin{gathered}
\stackrel{\circ}{A}_{i}, \stackrel{\circ}{\Phi} \in C^{k}, \\
\stackrel{\circ}{B}_{i}=\varepsilon_{i j k}\left(\partial_{j} \stackrel{\circ}{A}_{k}+\stackrel{\circ}{A}_{j} \stackrel{\circ}{k}_{k}\right) \in H_{k+1}, \\
\stackrel{\circ}{\pi}_{i}=\partial_{i} \stackrel{\circ}{\Phi}+\stackrel{\circ}{A}_{i} \stackrel{\circ}{\Phi} \in H_{k+1}, \\
\partial_{i} \stackrel{\circ}{A}_{j} \in H_{k-1}, \quad\left(|\stackrel{\circ}{\Phi}|^{2}-1\right) \in H_{k},
\end{gathered}
$$

for some specified $k \geqq 2$. These conditions are compatible with the magnetic charge associated with $\AA$

$$
\stackrel{\circ}{g}=\oint_{\infty} \stackrel{\circ}{\Phi} \cdot \stackrel{\circ}{B_{i}} d x^{i}
$$


and the topological winding number associated with $\Phi$, i.e. for a triplet

$$
\stackrel{\circ}{n}=\frac{1}{8 \pi} \oint_{\infty} \varepsilon_{i j k} \partial_{j} \stackrel{\circ}{\Phi}_{(a)} \partial_{k} \stackrel{\circ}{\Phi}_{(b)} \varepsilon_{a b c} \stackrel{\circ}{(c)}_{(c)} d x^{i}
$$

being non-zero. Although we are mainly interested in topologically nontrivial finite-energy configurations (magnetic monopoles), our proof covers topologically trivial configurations in topologically trivial and nontrivial models with spontaneous symmetry breaking as well.

\section{Local Existence Proof}

Following Segal we will work the local existence proof in the temporal gauge $A_{0}=0$. We will split $F_{\mu \nu}$ in the standard way,

$$
\begin{gathered}
E_{i}=F_{0 i}=\partial_{0} A_{i}, \\
B_{i}=\varepsilon_{i j k}\left(\partial_{j} A_{k}+A_{j} A_{k}\right) .
\end{gathered}
$$

We wish to make a further splitting, because we wish to write

$$
A_{i}(t, \mathbf{x})=\AA_{i}(\mathbf{x})+a_{i}(t, \mathbf{x}),
$$

where $\AA_{i}(\mathbf{x})$ is the static background with slow fall-off (with $\left.\AA_{0}=0\right), a_{i}(t, \mathbf{x})$ is the time-varying field with fast fall-off. Having defined

$$
\stackrel{\circ}{B}_{i}=\varepsilon_{i j k}\left(\partial_{j} \stackrel{\AA}{A}_{k}+\stackrel{\AA}{A}_{j} \stackrel{\circ}{k}_{k}\right) \text {, }
$$

we also define

$$
b_{i}=B_{i}-\stackrel{\circ}{B}_{i}=\varepsilon_{i j k}\left(\partial_{j} a_{k}+a_{j} a_{k}+\stackrel{\circ}{A}_{j} a_{k}+a_{j} \stackrel{\circ}{A}_{k}\right),
$$

and of course

$$
e_{i}=E_{i}=\partial_{0} a_{i} .
$$

The natural splitting of the Higgs field follows from writing

$$
\Phi(t, \mathbf{x})=\stackrel{\circ}{\Phi}(\mathbf{x})+\varphi(t, \mathbf{x}) .
$$

In general we have for the first derivative of $\Phi$,

$$
\begin{gathered}
\pi=D_{0} \Phi=\partial_{0} \Phi, \\
\pi_{i}=D_{i} \Phi=\partial_{i} \Phi+A_{i} \Phi .
\end{gathered}
$$

We now define

$$
\begin{gathered}
\psi=\partial_{0} \varphi=\pi, \\
\psi_{i}=\pi_{i}-\stackrel{\circ}{\pi}_{i}=\partial_{i} \varphi+a_{i} \varphi+a_{i} \stackrel{\circ}{\Phi}+\stackrel{\circ}{A}_{i} \varphi .
\end{gathered}
$$

The initial data (at a fixed time $t=0$ ) we will specify for the Yang-Mills-Higgs field will consist of the static background fields $\left(\AA_{i}, \Phi\right)$ and a sextuplet of fields

$$
\left(a_{i}, e_{i}, b_{i}, \varphi, \psi, \psi_{i}\right)=u(t, \mathbf{x}) \text {. }
$$


Following Ginibre and Velo [3] [their Eqs. (2.4) and (2.5)], the dynamical equations now read

$$
\partial_{0} u(t)=T u(t)+K(u(t))+\stackrel{\circ}{K}(u(t))
$$

with

$$
\begin{aligned}
& T=\left(\begin{array}{cc}
T_{a} & 0 \\
0 & T_{\varphi}
\end{array}\right), \\
& T_{a}=\left(\begin{array}{ccc}
0 & 1 & 0 \\
0 & 0 & R_{a} \\
0 & -R_{a}^{*} & 0
\end{array}\right), \quad T_{\varphi}=\left(\begin{array}{ccc}
0 & 1 & 0 \\
0 & 0 & R \\
0 & -R^{*} & 0
\end{array}\right), \\
& \left(R_{a}\right)_{i j}=\varepsilon_{i j k} \partial_{k}, \quad R=-\nabla, \\
& K=\left(0,-\varepsilon_{i j k}\left[a_{j}, b_{k}\right]-2 \operatorname{Re}\left\langle\psi_{i}, \theta_{a} \varphi\right\rangle \theta_{a},\right. \\
& \left.\varepsilon_{i j k}\left(a_{j} e_{k}+a_{j} a_{k}\right), 0, a_{i} \psi_{i}-|\varphi|^{2} \varphi, e_{i} \varphi+a_{i} \psi\right),
\end{aligned}
$$

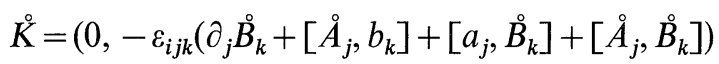

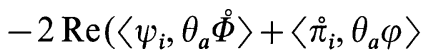

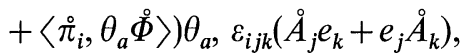

$$
\begin{aligned}
& 0, \partial_{i} \tilde{\pi}_{i}+\stackrel{\AA}{A}_{i} \psi_{i}+a_{i} \tilde{\pi}_{i}-2 \stackrel{\Phi}{ }|\varphi|^{2}
\end{aligned}
$$

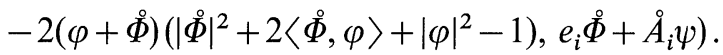

These, by themselves, do not constitute the Yang-Mills-Higgs field equations. We must add three constraints to them

$$
\begin{aligned}
& b_{i}-\varepsilon_{i j k}\left(\partial_{j} a_{k}+a_{j} a_{k}+\AA_{j} a_{k}+a_{j} \AA_{k}\right)=0, \\
& \psi_{i}-\partial_{i} \varphi-a_{i} \varphi-a_{i} \stackrel{\circ}{\Phi}-\AA_{i} \varphi=0, \\
& \partial_{i} e_{i}+\left[a_{i}, e_{i}\right]+2 \operatorname{Re}\left\langle\psi, \theta_{a} \varphi\right\rangle \theta_{a} \\
& +\left[\stackrel{\AA}{A}_{i}, e_{i}\right]+2 \operatorname{Re}\left\langle\psi, \theta_{a} \stackrel{\oplus}{\Phi}\right\rangle \theta_{a}=\mathcal{O} .
\end{aligned}
$$

These equations are essentially equivalent to those of Ginibre and Velo. The only difference is that their variables are

$$
\left(A_{i}, E_{i}, B_{i}, \Phi, \pi, \pi_{i}\right) \text { not }\left(a_{i}, e_{i}, b_{i}, \varphi, \psi, \psi_{i}\right) .
$$

With the change due to subtracting the background, we still have that the linear operator $T$ is identical to theirs, and our $K$ operator is identical to their non-linear term. The only change is the extra $K$ term, where we accumulate all terms which depend on the background.

We wish to apply the Segal existence theorem to (3.13). This theorem will not deal directly with (3.13), but with the associated integral equation

$$
u(t)=U(t) u_{0}+\int_{0}^{t} d \tau U(t-\tau) K_{\mathrm{tot}}(u(t))
$$

where

$$
K_{\text {tot }}=K+\stackrel{\circ}{K}
$$


and where $U$ is the transformation generated by the linear operator $T[(3.14)$ and (3.15)].

The first thing we have to do is to choose a Banach space $B$ in which we want $u(t)$ to lie. In this case the obvious choice is

$$
B_{k}=H_{k}\left(\mathbb{R}^{3}\right) \times H_{k} \times \ldots \times H_{k},
$$

where $k$ is some positive integer. We now show that $U$ is very well behaved on $B$.

Lemma 2.1 of Ginibre and Velo give us the desired result

Lemma 3.1. For any $k, U(t)$ is a (bounded) strongly continuous one parameter group in $B_{k}$ and for any $t \in \mathbb{R}, u_{0} \in B_{k}, U(t)$ satisfies the estimate

$$
\begin{gathered}
\left\|U(t) u_{0}\right\| \leqq \mu(t)\left\|u_{0}\right\|, \\
\mu(t)=\left\{1+\frac{1}{2}|t|\left(|t|+\left(t^{2}+4\right)^{1 / 2}\right)\right\}^{1 / 2} .
\end{gathered}
$$

We next have to look at $K_{\text {tot }}$ and show that $K_{\text {tot }}$ maps $B$ into itself. To do this we choose $k \geqq 2$. This allows us to use the fact that $H_{k}\left(\mathbb{R}^{3}\right)$ is a Banach algebra for $k \geqq 2$. There are no derivatives in $K$, only terms like $a_{i} e_{j}$. Since $a_{j} \in H_{k}$ and $e_{j} \in H_{k} \Rightarrow a_{i} e_{j} \in H_{k}$, obviously $K$ maps $B \rightarrow B$. In $K^{\circ}$ there are two derivative terms $\partial_{j} B$ and $\partial_{i} \stackrel{\circ}{\pi}$. We put as part of the initial conditions that $B, \AA \in H^{\circ} \in H_{k+1}$. The other terms are of the kind $\AA_{i} e_{j}$. If we demand $\AA_{i} \in C^{k}$, then $e_{j} \in H_{k} \Rightarrow \AA_{i} e_{j} \in H_{k}$. The only other term that needs special treatment is $\Phi^{2}-1 \in H_{k}$. It then follows that the operator $K_{\text {tot }}$ maps $B$ into itself.

The second property that we need for $K_{\text {tot }}$ is that it satisfy a Lipschitz condition

$$
\left\|K_{\text {tot }}\left(u_{1}\right)-K_{\text {tot }}\left(u_{2}\right)\right\| \leqq C\left(\left\|u_{1}\right\|,\left\|u_{2}\right\|\right)\left\|u_{1}-u_{2}\right\|,
$$

where $C$ is an increasing finite real function, and $\|\cdot\|$ is the $\left(H_{k}\right)^{6}$ norm. This again follows from the Banach Algebra property of $H_{k}$ for $k \geqq 2$. All the terms in $K$ are of the form $a b$ except for the $\varphi^{3}$ term. The $a b$ term leads to

$$
a_{1} b_{1}-a_{2} b_{2}=a_{1}\left(b_{1}-b_{2}\right)-b_{2}\left(a_{1}-a_{2}\right)
$$

and

$$
\left\|a_{1}\left(b_{1}-b_{2}\right)\right\|_{H_{k}} \leqq C_{0}\left\|a_{1}\right\|_{H_{k}}\left\|b_{1}-b_{2}\right\|_{H_{k}} \text {. }
$$

The $\varphi^{3}$ term gives

$$
\left\|\varphi_{1}^{3}-\varphi_{2}^{3}\right\|=\left(\varphi_{1}-\varphi_{2}\right)\left(\varphi_{1}^{2}+\varphi_{1} \varphi_{2}+\varphi_{2}^{2}\right)
$$

and

$$
\begin{aligned}
& \left\|\left(\varphi_{1}^{2}+\varphi_{1} \varphi_{2}+\varphi_{2}^{2}\right)\left(\varphi_{1}-\varphi_{2}\right)\right\|_{\boldsymbol{H}_{k}} \leqq C_{0}\left\|\varphi_{1}^{2}+\varphi_{1} \varphi_{2}+\varphi_{2}^{2}\right\|_{\boldsymbol{H}_{k}}\left\|\varphi_{1}-\varphi_{2}\right\|_{H_{k}} \\
& \quad \leqq C_{1}\left(\left\|\varphi_{1}\right\|+\left\|\varphi_{2}\right\|\right)^{2}\left\|\varphi_{1}-\varphi_{2}\right\|_{\boldsymbol{H}_{k}} .
\end{aligned}
$$

The terms in $K$ are of the form $\AA b$ which gives $\AA\left(b_{1}-b_{2}\right)$ and

$$
\left\|\stackrel{\mathrm{A}}{ }\left(b_{1}-b_{2}\right)\right\|_{H_{k}} \leqq\|\AA\|_{C^{k}}\left\|b_{1}-b_{2}\right\|_{H_{k}},
$$

together with $\Phi \varphi^{2}$ which gives $\Phi\left(\varphi_{1}^{2}-\varphi_{2}^{2}\right)$ and satisfies

$$
\left\|\stackrel{\Phi}{(}\left(\varphi_{1}^{2}-\varphi_{2}^{2}\right)\right\|_{H_{k}} \leqq C_{2}\|\stackrel{\Phi}{ }\|_{C^{k}}\left(\left\|\varphi_{1}\right\|+\left\|\varphi_{2}\right\|\right)\left\|\varphi_{1}-\varphi_{2}\right\|_{H_{k}} .
$$

All these together combine to prove (3.24). 
The Lipshitz condition on $K_{\text {tot }}$ and the fact that $U(t)$ is a (semi)group is all that we need to apply the Segal existence theory. This immediately gives us that there exists a unique solution to (3.21) for some finite time interval $[-T, T]$ and that $u(t)$ for any $t \in[-T, T]$ belongs to $H_{k}$.

To go from the integral Eq. (3.21) to the differential equation (3.13), we have to show that $K_{\text {tot }}$ is a $C^{1}$ map. This is quite straightforward. Since $K_{\text {tot }}$ is a third order polynomial in $u$ (the $\varphi^{3}$ term), the Frechet derivative of $K_{\text {tot }}$ is quadratic in $u$. We now just repeat our proof of the Lipschitz condition for this simpler case. Analogously, we can prove that $K_{\text {tot }}$ is a $C^{\infty}$ map since all derivatives higher than third order vanish.

As we remarked earlier, the differential Eq. (3.13) is not equivalent to the YangMills-Higgs equations. We need to also satisfy the constraints (3.18), (3.19), (3.20). This is quite straightforward. We choose the initial data at $t=0$ so as to satisfy the constraints. The dynamical Eq. (3.13) is consistent with the constraints and automatically propagates them (see Proposition 2.3 of Ginibre and Velo). Thus we have our desired local existence proof.

\section{A Priori Bounds}

To expand the local proof to a global existence proof we will switch over to imitate the global existence proof of Eardley and Moncrief [4]. The key idea in their analysis is to obtain an a priori bound on $F_{\mu \nu}$ and $D_{\mu} \Phi$.

We start off with regular initial data and use the local existence proof to demonstrate the existence of a classical solution on a patch of spacetime. (To guarantee the smoothness Eardley and Moncrief want for working in Cronström gauge we impose the necessary smoothness conditions on the initial value data.) To extend this to a global solution we have to show that nothing goes bad at the boundary. This requires showing that the $H_{2}$ norms of $\left(a_{i}, e_{i}, b_{i}, \varphi, \psi, \psi_{i}\right)$ do not blow up. This means that we extend beyond the boundary and so continue.

Just as with the local Ginibre and Velo proof [3], we need only make minor modifications to the global Eardley and Moncrief proof to accommodate the extra $\AA$ and $\Phi$ terms. The first point of importance is that we still have a finite conserved energy $E_{0}=\int_{\mathbb{R}^{3}} \varepsilon d^{3} x$, where

$$
\varepsilon=\frac{1}{2}\left(E^{2}+B^{2}\right)+\pi \cdot \pi+D_{i} \Phi \cdot D^{i} \Phi+\left(|\Phi|^{2}-1\right)^{2}>0 .
$$

This conserved energy can be derived from an energy-momentum tensor $T^{\mu v}$ given by

$$
T^{\mu v}=\operatorname{Tr}\left\{F^{\mu \alpha} F_{\alpha}^{v}-\frac{1}{4} \eta^{\mu v} F_{\alpha \beta} F^{\alpha \beta}\right\}+2 D^{\mu} \Phi \cdot D^{v} \Phi-\eta^{\mu v} D_{\alpha} \Phi \cdot D^{\alpha} \Phi-\eta^{\mu v}\left(|\Phi|^{2}-1\right)^{2},
$$

which satisfies $\partial_{v} T^{\mu v}=0$.

Let us choose a point $p$ in the domain of local existence and shift coordinates so that $p$ becomes the origin of coordinates. The initial slice now is labeled $t=-t_{0}$. Consider the back light cone $K_{p}$ from $p$ to the original surface. Call the solid sphere where the light-cone intersects the original surface $B_{p}$. Now consider the 
conservation of energy on the set which is the interior of $K_{p}$ (call it $\tilde{K}_{p}$ )

$$
0=\int_{\widetilde{K}_{p}} \partial_{v} T_{0}^{v} d^{4} x=\int_{K_{p}} T_{0}^{\nu} \cdot n+\int_{B_{p}} T_{0}^{v} \cdot n
$$

(on using the Gauss theorem). This, when written out, is of the form

$$
\begin{aligned}
& \int_{K_{p}} r^{2} d r d \Omega\left\{\frac { 1 } { 2 } \operatorname { T r } \left[\frac{3}{4}(\hat{\ell} \cdot F \cdot \hat{m})^{2}+\left(\hat{\ell} \cdot F \cdot \hat{e}_{A}\right)^{2}\right.\right. \\
&\left.+\frac{1}{2}\left(\hat{e}_{A} \cdot F \cdot \hat{e}_{B}\right)^{2}\right]+\left(|\Phi|^{2}-1\right)^{2} \\
&+\left[D_{\hat{e}} \Phi \cdot D_{\hat{e}} \Phi+D_{\hat{e}_{A}} \Phi \cdot D_{\hat{e}_{A}} \Phi\right] \\
&= \int_{B_{P}} \varepsilon d^{3} x<E_{0},
\end{aligned}
$$

where

$$
\begin{gathered}
\hat{\ell}=-\partial / \partial t+\partial / \partial r, \quad \hat{m}=\partial / \partial t+\partial / \partial r, \\
\hat{e}=\left(\frac{1}{r} \partial / \partial \vartheta, \frac{1}{r \sin \vartheta} \partial / \partial \varphi\right) .
\end{gathered}
$$

The integral along the light-cone is a set of quadratic (positive) terms. Therefore we have that each one individually is bounded by the finite total energy $E_{0}$, i.e.

and so on.

$$
\begin{gathered}
\int_{K_{p}} r^{2} d r d \Omega \operatorname{Tr}(\hat{\ell} \cdot F \cdot \hat{m})^{2}<E_{0}, \\
\int_{K_{p}} r^{2} d r d \Omega\left(|\Phi|^{2}-1\right)^{2}<E_{0},
\end{gathered}
$$

The next stage is to realise that the Yang-Mills equations can be manipulated to give us wave equations for $F$ and $D \Phi$ :

$$
\begin{aligned}
\eta^{\mu v} \partial_{\mu} \partial_{v} F_{\alpha \beta}= & -2 \partial_{\gamma}\left[A^{\gamma}, F_{\alpha \beta}\right]+\left[\partial_{\gamma} A^{\gamma}, F_{\alpha \beta}\right] \\
& -\left[A^{\gamma},\left[A_{\gamma}, F_{\alpha \beta}\right]\right]+2\left[F_{\alpha}^{\gamma}, F_{\gamma \beta}\right]+2\left(\left(F_{\alpha \beta} \Phi\right) \cdot \theta_{a} \Phi\right) \theta_{a} \\
& +2\left(\left(D_{\beta} \Phi\right) \cdot \theta_{a}\left(D_{\alpha} \Phi\right)-2\left(D_{\alpha} \Phi\right) \cdot \theta_{a}\left(D_{\beta} \Phi\right)\right) \theta_{a}=: \varrho_{\alpha \beta}, \\
\eta^{\mu \nu} \partial_{\mu} \partial_{v}\left(D_{\alpha} \Phi\right)=- & 2 \partial_{\mu}\left(A^{\mu} D_{\alpha} \Phi\right)+\left(\partial_{\mu} A^{\mu}\right) D_{\alpha} \Phi-A_{\mu} A^{\mu} D_{\alpha} \Phi \\
+ & 2\left(\left(D_{\alpha} \Phi\right) \cdot \theta_{a} \Phi\right) \theta_{a} \Phi-2 F_{\alpha}^{\mu} D_{\mu} \Phi+4 D_{\alpha}\left\{\Phi\left(|\Phi|^{2}-1\right)\right\}=: \varrho_{\alpha} .
\end{aligned}
$$

The right hand side of each of these equations can be regarded as a source term for a wave equation. This allows us to write $F_{\alpha \beta}$ at $p$ as the average value of $F_{\alpha \beta}$ on the boundary of $B_{p}$ plus a term arising from the integral of the source along $K_{p}$. In other words

$$
F_{\alpha \beta}(p)=\frac{1}{4 \pi} \int_{S^{2}} d \Omega\left(r_{0} m^{\mu} \partial_{\mu} F_{\alpha \beta}+F_{\alpha \beta}\right)-\frac{1}{4 \pi} \int_{K_{p}} r d r d \Omega \varrho_{\alpha \beta},
$$

where $S_{2}$ is the sphere of radius $r=r_{0}=-t_{0}$ on the original slice. Equivalently we have

$$
D_{\mu} \Phi(p)=\frac{1}{4 \pi} \int_{S^{2}} d \Omega\left(r_{0} m^{2} \partial_{\alpha} D_{\mu} \Phi+D_{\mu} \Phi\right)-\frac{1}{4 \pi} \int_{K_{p}} r d r d \Omega \varrho_{\mu} .
$$


There is no difficulty in handling the two-sphere integral and showing that it finite. The real problem arises from the light-cone integral, and there we need to use the bounds obtained from the energy conservation equation. One useful bound that can be obtained is

$$
\begin{aligned}
\int_{K_{p}}\left(|\Phi|^{2}-1\right)^{2}<E_{0} & \Rightarrow \int_{K_{p}}\left|\left(|\Phi|^{2}-1\right)\right|<\sqrt{C E_{0}} t_{0}^{3 / 2} \\
& \Rightarrow \int_{K_{p}}|\Phi|^{2}<\sqrt{C E_{0}} t_{0}^{3 / 2}+C t_{0}^{3}
\end{aligned}
$$

(where $C t_{0}^{3}$ is the volume of the cone) and also

$$
\int_{K_{p}}|\Phi|^{4}<E_{0}+2 \sqrt{C E_{0}} t_{0}^{3 / 2}+3 C t_{0}^{3} .
$$

The only real problem we have in going from Eardley-Moncrief to our spaces is the fact that for us such functions as

$$
\int_{\mathbb{R}^{3}}|\Phi|^{2} d^{3} x
$$

are not finite.

However, in deriving the a priori bounds on $F$ and $D \Phi$ we need never integrate over $\mathbb{R}^{3}$ only over $K_{p}$, where the relevant integrals are finite and so the proof goes through unchanged. Thus we also have that $\|F\|_{L^{\infty}}$ and $\left\|D_{\mu} \Phi\right\|_{L^{\infty}}$ remain finite.

\section{Bounds on Everything Else}

Given that $\|F\|_{L^{\infty}}$ and $\left\|D_{\mu} \Phi\right\|_{L^{\infty}}$ remain finite in the local range of existence of a solution, we now need to show that the $\mathrm{H}_{2}$ norms of $\left(a_{i}, e_{i}, b_{i}, \varphi, \psi, \psi_{i}\right)$ also remain finite. We begin by showing that the $L_{2}$ norms remain finite. Let us begin with

$$
\frac{d}{d t}\left(\|\varphi\|_{L_{2}}\right)^{2}=2 \int_{\mathbb{R}^{3}} \psi \cdot \varphi \leqq 2\left|\int_{\mathbb{R}^{3}} \psi^{2}\right|^{1 / 2}\left|\int_{\mathbb{R}^{3}} \phi^{2}\right|^{1 / 2} \leqq \sqrt{2 E_{0}}\|\phi\|_{L_{2}},
$$

which implies

$$
\|\varphi(t)\|_{L^{2}} \leqq\|\varphi(0)\|_{L_{2}}+\sqrt{2 E_{0}} t,
$$

and so $\|\varphi\|_{L_{2}}$ remains finite. Next

$$
\frac{d}{d t}\left(\left\|a_{i}\right\|_{L_{2}}\right)^{2}=2 \int_{\mathbb{R}^{3}} a_{i} \cdot e_{i} \leqq \sqrt{2 E_{0}}\left\|a_{i}\right\|_{L_{2}} .
$$

The $L_{2}$ norms of $e_{i}, \psi, B_{i}=b_{i}+\stackrel{\circ}{i}_{i}$ and $D_{i} \Phi=\psi_{i}+\stackrel{\circ}{i}_{i}$ do not blow up from energy conservation. Because $B_{i}$ and $\dot{\pi}_{i}$ are constant and belong to $L_{2}$, the $L_{2}$ norms of $b_{i}$ and $\psi_{i}$ cannot blow up.

The next stage is to integrate the equations

$$
\frac{\partial}{\partial t} a_{i}=e_{i}, \quad \frac{\partial}{\partial t} \varphi=\psi
$$

to give

$$
\left\|a_{i}\right\|_{L^{\infty}} \leqq\left\|a_{i}(0)\right\|_{L^{\infty}}+\int_{0}^{t} d s\left\|e_{i}(s)\right\|_{L^{\infty}}<\infty
$$


and

$$
\|\varphi\|_{L^{\infty}} \leqq\|\varphi(0)\|_{L^{\infty}}+\int_{0}^{t} d s\|\psi(s)\|_{L^{\infty}} .
$$

The final inequality follows from the fact that $e_{i}(s)$ is part of $F_{\alpha \beta}$ and $\psi(s)$ is part of $D \Phi$, both of which were shown to remain finite in Sect. IV.

We have that

$$
b_{i}=B_{i}-\stackrel{\circ}{B}_{i}=\varepsilon_{i j k}\left(\partial_{j} a_{k}+a_{j} a_{k}+\AA_{j} a_{k}+a_{j} \stackrel{\AA}{k}_{k}\right),
$$

and

$$
\psi_{i}=\pi_{i}-\stackrel{\circ}{\pi}_{i}=\partial_{i} \varphi+a_{i} \varphi+a_{i} \dot{\Phi}+\stackrel{\circ}{A}_{i} \varphi
$$

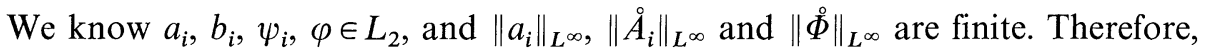
$\partial_{i} \varphi \in L_{2}$ and $\varepsilon_{i j k} \partial_{j} a_{k} \in L_{2}$ hold. Further $\left\|\psi_{i}\right\|_{L^{\infty}},\left\|\partial_{i} \varphi\right\|_{L^{\infty}},\left\|b_{i}\right\|_{L^{\infty}}$, and $\left\|\varepsilon_{i j k} \partial_{j} a_{k}\right\|_{L^{\infty}}$ are all finite. Finally the third constraint

$$
\partial_{i} e_{i}=-\left[a_{i}, e_{i}\right]-2 \operatorname{Re}\left\langle\psi, \theta_{a} \varphi\right\rangle \theta_{a}-\left[\stackrel{\circ}{A}_{i}, e_{i}\right]-2 \operatorname{Re}\left\langle\psi, \theta_{a} \stackrel{\oplus}{\Phi}\right\rangle \theta_{a}
$$

also gives us $\partial_{i} e_{i} \in L_{2}$, and $\left\|\partial_{i} e_{i}\right\|_{L^{\infty}}$ finite.

The next stage is to show that $\left(a_{i}, e_{i}, b_{i}, \varphi, \psi, \psi_{i}\right) \in H_{1}$. This means

$$
\partial_{i} a_{j}, \partial_{i} e_{j}, \partial_{i} b_{j}, \partial_{i} \varphi, \partial_{i} \psi, \partial_{i} \psi_{j} \in L_{2} .
$$

Let us start with $\partial_{i} a_{j}$ and consider

$$
\mathscr{E}_{0}=-\frac{1}{2} \operatorname{Tr} \int_{\mathbb{R}^{3}}\left[e_{i} \cdot e_{i}+\left(\partial_{i} a_{j}\right)\left(\partial_{i} a_{j}\right)\right] d^{3} x .
$$

Now

$$
\begin{aligned}
\frac{d}{d t} \mathscr{E}_{0}= & -\operatorname{Tr} \int\left[e _ { i } \left\{\varepsilon_{i j k} \partial_{k} b_{i}-\varepsilon_{i j k}\left[a_{j}, b_{k}\right]\right.\right. \\
& -2 \operatorname{Re}\left\langle\psi_{i}, \theta_{a} \varphi\right\rangle \theta_{a}+\varepsilon_{i j k}\left(\partial_{k} \dot{B}_{j}-\left[\stackrel{\circ}{A}_{j}, b_{k}\right]\right. \\
& -\left[a_{j}, \stackrel{\circ}{B}_{k}\right]-\left[\stackrel{\circ}{A}_{j}, \stackrel{\circ}{B}_{k}\right]-2 \operatorname{Re}\left(\left\langle\psi_{i}, \theta_{a} \varphi\right\rangle\right. \\
& \left.\left.\left.+\left\langle\stackrel{\circ}{\pi}_{i}, \theta_{a} \varphi\right\rangle+\left\langle\stackrel{\circ}{\pi}_{i}, \theta_{a} \dot{\Phi}\right\rangle\right) \theta_{a}\right\}+\partial_{i} a_{j} \partial_{i} e_{j}\right] d^{3} x .
\end{aligned}
$$

All the terms in the expression above involving background fields are clearly finite and the two terms $e_{i} \varepsilon_{i j k}\left[a_{j}, b_{k}\right]$ and $e_{i} \operatorname{Re}\left\langle\psi_{i}, \theta_{a} \varphi\right\rangle \theta_{a}$ are finite because $\left\|e_{i}\right\|_{L^{\infty}}$, $\left\|a_{j}\right\|_{L_{2}},\left\|b_{k}\right\|_{L_{2}},\left\|\psi_{i}\right\|_{L_{2}},\|\varphi\|_{L_{2}}<\infty$.

This leaves only

$$
\begin{aligned}
\int \operatorname{Tr}\left\{e_{i} \varepsilon_{i j k} \partial_{k} b_{i}+\partial_{i} a_{j} \partial_{i} e_{j}\right\}=\int & \operatorname{Tr}\left\{e _ { i } \partial _ { k } \left(\partial_{k} a_{i}-\partial_{i} a_{k}+\left[a_{k}, a_{i}\right]\right.\right. \\
& \left.\left.+\left[\stackrel{\AA_{k}}{A_{k}} a_{i}\right]+\left[a_{k}, \AA_{i}\right]\right)+\partial_{i} a_{j} \partial_{i} e_{j}\right\} .
\end{aligned}
$$

The first and last terms combine to form $\partial_{k}\left(e_{i} \partial_{k} a_{i}\right)$ and so integrate to zero. The second term can be written as

$$
\partial_{i} e_{i} \partial_{k} a_{k}-\partial_{i}\left(e_{i} \partial_{i} a_{k}\right)
$$


Now

$$
\int \partial_{i} e_{i} \partial_{k} a_{k} \leqq\left\|\partial_{i} e_{i}\right\|_{L_{2}}\left\|\partial_{k} a_{k}\right\|_{L_{2}} \leqq C \sqrt{\mathscr{E}_{0}}
$$

since $\left\|\partial_{i} e_{i}\right\|_{L_{2}}$ is finite. The total divergence can again be ignored. The three commutator terms fall in line, the worst being

$$
\int e_{i} a_{k} \partial_{k} a_{i} \leqq\left\|e_{i}\right\|_{L^{\infty}}\left\|a_{k}\right\|_{L_{2}}\left\|\partial_{k} a_{i}\right\|_{L_{2}} \leqq C_{1} \sqrt{\mathscr{E}_{0}}
$$

Hence we have

$$
\frac{d}{d t} \mathscr{E}_{0}=C_{2}+C_{3} \sqrt{\mathscr{E}_{0}}
$$

which implies that $\mathscr{E}_{0}$ remains finite and hence $\partial_{i} a_{k} \in L_{2}$.

To show that $e_{i}, b_{i}, \psi$, and $\psi_{i}$ belong to $H_{1}$ we have to show that

$$
\mathscr{E}_{1}=\frac{1}{2} \int\left\{\left(\partial_{j} e_{i}\right)\left(\partial_{j} e_{i}\right)+\left(\partial_{j} b_{i}\right)\left(\partial_{j} b_{i}\right)+\left(\partial_{i} \psi\right)\left(\partial_{i} \psi\right)+\left(\partial_{i} \psi_{j}\right)\left(\partial_{i} \psi_{j}\right)\right\} d^{3} x
$$

remains finite. To show this we calculate

$$
\begin{aligned}
& \frac{d}{d t} \mathscr{E}_{1}=\int\left\{\partial _ { j } e _ { i } \partial _ { j } \left[\varepsilon_{i m n} \partial_{n} b_{m}-\varepsilon_{i m n}\left[a_{m}, b_{n}\right]\right.\right. \\
& -2 \operatorname{Re}\left\langle\psi_{i}, \theta_{a} \varphi\right\rangle \theta_{a}-\varepsilon_{i m n}\left(\partial_{m} \stackrel{\circ}{B}_{n}+\left[a_{m}, \stackrel{\circ}{B}_{n}\right]\right. \\
& \left.+\left[\stackrel{\circ}{A}_{m}, b_{n}\right]+\left[\stackrel{\circ}{A}_{m}, \stackrel{\circ}{B}_{n}\right]\right)-2 \operatorname{Re}\left(\left\langle\psi_{i}, \theta_{a} \check{\Phi}^{\circ}\right\rangle\right. \\
& +\left\langle\stackrel{\circ}{\pi}_{i}, \theta_{a} \varphi\right\rangle+\left\langle\stackrel{\circ}{\pi}_{i}, \theta_{a} \Phi\right) \theta_{a} \\
& +\partial_{j} b_{i} \partial_{j}\left[\varepsilon_{i m n} \partial_{m} e_{n}+\varepsilon_{i m n}\left[a_{m}, e_{n}\right]\right. \\
& \left.\left.+\varepsilon_{i m n}\left[\AA_{m}, e_{n}\right]\right]+\left(\psi / \psi_{i}-\text { terms }\right)\right\} \text {. }
\end{aligned}
$$

The two leading terms form a total divergence $\partial_{n}\left\{\partial_{j} e_{i} \varepsilon_{i m n} \partial_{j} b_{m}\right\}$ and so can be ignored. The next terms to be considered are

$$
\int \partial_{j} e_{i} \partial_{j} a_{m} b_{n} \leqq\left\|b_{n}\right\|_{L^{\infty}}\left\|\partial_{j} e_{i}\right\|_{L_{2}}\left\|\partial_{j} a_{m}\right\|_{L_{2}} \leqq C \sqrt{\mathscr{E}_{1}},
$$

and

$$
\int a_{m} \partial_{j} e_{i} \partial_{j} b_{n} \leqq\left\|a_{m}\right\|_{L^{\infty}}\left\|\partial_{j} e_{i}\right\|_{L_{2}}\left\|\partial_{j} b_{n}\right\|_{L_{2}} \leqq C \mathscr{E}_{1} .
$$

The terms $\partial_{j} e_{i} \partial_{j} \psi_{i} \varphi$ and $\psi_{i} \partial_{j} e_{i} \partial_{j} \varphi$ can be handled in the same way.

All the terms involving the background fields are quite straightforward. We only need to use

$$
\partial_{i} \partial_{j} \stackrel{\circ}{B}_{m}, \partial_{j} \stackrel{\AA}{A}_{m} \in L_{2} ; \quad \stackrel{\circ}{A}_{m}, \stackrel{\circ}{B}_{m} \in L^{\infty}
$$

to get terms proportional to $\sqrt{\mathscr{E}_{1}}$ and to $\mathscr{E}_{1}$. We finally get

$$
\frac{d}{d t} \mathscr{E}_{1} \leqq C_{1} \sqrt{\mathscr{E}_{1}}+C_{2} \mathscr{E}_{1}
$$

which shows that $\mathscr{E}_{1}$ remains finite, and hence

$$
\left\|\partial_{j} e_{i}\right\|_{L_{2}},\left\|\partial_{j} b_{i}\right\|_{L_{2}},\left\|\partial_{i} \psi\right\|_{L_{2}},\left\|\partial_{i} \psi_{j}\right\|_{L_{2}}
$$


remain finite. These can now be inserted into the constraints to force $\varepsilon_{i j k} \partial_{j} a_{k}, \partial_{i} \varphi$, and $\partial_{i} e_{i}$ to belong to $H_{1}$.

The next step is to show that $\partial_{j} a_{k}$ itself belongs to $H_{1}$, or equivalently that $\partial_{i} \partial_{j} a_{k}$ belongs to $L_{2}$. This is the same as showing that

$$
\mathscr{E}_{1}^{\prime}=\frac{1}{2} \int\left\{\left(\partial_{j} e_{i}\right)\left(\partial_{j} e_{i}\right)+\left(\partial_{i} \partial_{j} a_{k}\right)\left(\partial_{i} \partial_{j} a_{k}\right)\right\}
$$

remains finite. To do this we calculate

$$
\frac{d}{d t} \mathscr{E}_{1}^{\prime}=\int\left\{\left(\partial_{j} e_{i}\right)\left(\partial_{j} \partial_{0} e_{i}\right)+\left(\partial_{i} \partial_{j} a_{k}\right)\left(\partial_{i} \partial_{j} e_{k}\right)\right\} .
$$

Substituting for $\partial_{0} e_{i}$ we get a total derivative plus

$$
\partial_{j}\left(\partial_{i} e_{i}\right) \partial_{j} \partial_{n} a_{n}-\left(\partial_{j} e_{i}\right) \partial_{j} \partial_{n}\left[a_{i}, a_{n}\right]+\ldots .
$$

On using $\partial_{i} e_{i} \in H_{1}$ we can bound the first of these terms by $C \sqrt{\mathscr{E}_{1}^{\prime}}$.

The second term requires some care. One part will be of the form

$$
\int a_{i} \partial_{j} e_{i} \partial_{j} \partial_{n} a_{n} \leqq\left\|a_{i}\right\|_{L^{\infty}}\left\|\partial_{j} e_{i}\right\|_{L_{2}}\left\|\partial_{j} \partial_{n} a_{n}\right\|_{L_{2}} \leqq C \mathscr{E}_{1}^{\prime} .
$$

The other parts, however, are of the form

$$
\partial_{j} e_{i} \partial_{j} a_{i} \partial_{n} a_{n} \text { or } \partial_{j} a_{i} \partial_{n} a_{i} \partial_{j} a_{n} .
$$

The trick is to remember that $\left\|F_{\mu \nu}\right\|_{L^{\infty}}$ is finite which implies $\left\|b_{i}\right\|_{L^{\infty}}$ finite which implies $\left\|\partial_{j} a_{i}-\partial_{i} a_{j}\right\|_{L^{\infty}}$ finite, and so we can replace $\partial_{j} \mathrm{a}_{i}$ with $\partial_{i} a_{j}$ to give

$$
\partial_{j} e_{i} \partial_{i} a_{j} \partial_{n} a_{n}=\partial_{i}\left[\partial_{j} e_{i} a_{j} \partial_{n} a_{n}\right]-a_{j} \partial_{n} a_{n} \partial_{j} \partial_{i} e_{i}-a_{j} \partial_{j} e_{i} \partial_{i} \partial_{n} a_{n} .
$$

The divergence is fine, the first term is finite because $\partial_{i} e_{i} \in H_{1}$ and the second term is proportional to $\sqrt{\mathscr{E}_{1}^{\prime}}$. Everything else is well behaved, so we finally get

$$
\left(a_{i}, \varphi\right) \in H_{2}, \quad\left(e_{i}, b_{i}, \psi, \psi_{i}\right) \in H_{1} .
$$

The last stage of the proof is to show that the $\mathrm{H}_{2} \times \mathrm{H}_{2}$ norm remains finite. The obvious thing to do is to write down the equivalent of $\mathscr{E}_{1}$, i.e.

$$
\begin{aligned}
\mathscr{E}_{2}= & \frac{1}{2} \int\left\{\left(\partial_{k} \partial_{j} e_{i}\right)\left(\partial_{k} \partial_{j} e_{i}\right)+\left(\partial_{k} \partial_{j} b_{i}\right)\left(\partial_{k} \partial_{j} b_{i}\right)+\left(\partial_{k} \partial_{j} \psi\right)\left(\partial_{k} \partial_{j} \psi\right)\right. \\
& \left.+\left(\partial_{k} \partial_{j} \psi_{i}\right)\left(\partial_{k} \partial_{j} \psi_{i}\right)\right\} d^{3} x
\end{aligned}
$$

and show $d \mathscr{E}_{2} / d t \leqq\left(\right.$ polynomial with constant coefficients in $\left.\mathscr{E}_{2}\right)$. Now

$$
\begin{aligned}
\frac{d}{d t} \mathscr{E}_{2}= & \int \partial_{k} \partial_{j} e_{i} \partial_{k} \partial_{j}\left[\varepsilon_{i m n} \partial_{n} b_{m}-\varepsilon_{i m n}\left[a_{m}, b_{n}\right]\right. \\
& -2 \operatorname{Re}\left\langle\psi_{i}, \theta_{a} \varphi\right\rangle \theta_{a}-\varepsilon_{i m n}\left(\partial_{m} \stackrel{\circ}{B}_{n}+\left[a_{m}, \stackrel{\circ}{n}_{n}\right]+\left[\check{A}_{m}, b_{n}\right]+\left[\stackrel{\leftrightarrow}{A}_{m}, \stackrel{\circ}{B}_{n}\right]\right) \\
& \left.-2 \operatorname{Re}\left(\left\langle\psi_{i}, \theta_{a} \stackrel{\circ}{\Phi}\right\rangle+\left\langle\stackrel{\circ}{\pi}_{i}, \theta_{a} \varphi\right\rangle+\left\langle\stackrel{\circ}{i}_{i}, \theta_{a} \Phi\right\rangle\right) \theta_{a}\right] \\
& +\partial_{k} \partial_{j} b_{i} \partial_{j} \partial_{k}\left[\varepsilon_{i m n} \partial_{m} e_{n}+\varepsilon_{i m n}\left[a_{m}, e_{n}\right]+\varepsilon_{i m n}\left[\stackrel{\circ}{A}_{m}, e_{n}\right]\right] \\
& +\left(\psi / \psi_{i}-\text { terms }\right) .
\end{aligned}
$$


Again, the leading terms form total divergences

$$
\partial_{n}\left[\varepsilon_{i m n} \partial_{k} \partial_{j} e_{i} \partial_{k} \partial_{j} b_{m}\right] \text { and } \partial_{i}\left[\partial_{k} \partial_{j} \psi \partial_{k} \partial_{j} \psi_{i}\right] \text {, }
$$

and so can be ignored. However when we look at the next term we run into difficulties. This is of the form

$$
\partial_{k} \partial_{j} e_{i} \varepsilon_{i m n} \partial_{k} \partial_{j}\left[a_{m}, b_{n}\right]
$$

The two terms

$$
\int \varepsilon_{i m n} b_{n} \partial_{k} \partial_{j} e_{i} \partial_{k} \partial_{j} a_{m} \leqq\left\|b_{n}\right\|_{L^{\infty}}\left\|\partial_{k} \partial_{j} e_{i}\right\|_{L_{2}}\left\|\partial_{k} \partial_{j} a_{m}\right\|_{L_{2}} \leqq C \sqrt{\mathscr{E}_{2}},
$$

and

$$
\int \varepsilon_{i m n} a_{m} \partial_{k} \partial_{j} e_{i} \partial_{k} \partial_{j} b_{n} \leqq\left\|a_{m}\right\|_{L^{\infty}}\left\|\partial_{k} \partial_{j} e_{i}\right\|_{L_{2}}\left\|\partial_{k} \partial_{j} b_{n}\right\|_{L_{2}} \leqq C \mathscr{E}_{2}
$$

offer no difficulty, but the third term $\int \varepsilon_{i m n} \partial_{k} \partial_{j} e_{i} \partial_{j} a_{m} \partial_{k} b_{n}$ has to be dealt with carefully. We know that it is bounded by

$$
\left\|\partial_{k} \partial_{j} e_{i}\right\|_{L_{2}}\left\|\partial_{j} a_{m} \partial_{k} b_{n}\right\|_{L_{2}} .
$$

We also know from the Sobolev multiplication theorem that $H_{1} \times H_{1}$ is uniformly embedded in $L_{2}$. Hence

$$
\left\|\partial_{j} a_{m} \partial_{k} b_{n}\right\|_{L_{2}} \leqq C_{0}\left\|\partial_{j} a_{m}\right\|_{H_{1}}\left\|\partial_{k} b_{n}\right\|_{H_{1}} .
$$

But of course $\left\|\partial_{j} a_{m}\right\|_{H_{1}}$ is finite and $\left\|\partial_{k} b_{n}\right\|_{H_{1}}$ is bounded by a finite number times $\sqrt{\mathscr{E}_{2}}$. Hence

$$
\int \varepsilon_{i m n} \partial_{k} \partial_{j} e_{i} \partial_{j} a_{m} \partial_{k} b_{n} \leqq C_{1} \sqrt{\mathscr{E}_{2}}+C_{2} \mathscr{E}_{2}
$$

All the other terms are well behaved. To deal with the terms which include the background terms we need slightly sharper conditions, on these, i.e.

$$
\stackrel{\circ}{B} \in H_{3}, \quad \stackrel{\circ}{\pi}_{i} \in H_{3}, \quad \partial_{j} \partial_{k} \AA \in L_{2},
$$

and we finally get

$$
\frac{d}{d t} \mathscr{E}_{2} \leqq C_{1}+C_{2} \sqrt{\mathscr{E}_{2}}+C_{3} \mathscr{E}_{2}
$$

This guarantees that $\mathscr{E}_{2}$ remains finite and hence that each of $\left(e_{i}, b_{i}, \psi, \psi_{i}\right)$ remain in $\mathrm{H}_{2}$ if originally there. We already know $a_{i}$ remains in $H_{2}$, and of course since $\psi_{i}=\partial_{i} \varphi+a_{i} \varphi+a_{i} \stackrel{\Phi}{\Phi}+\AA_{i} \varphi$, we know that $\varphi$ belongs to $H_{3}$. Hence we have that the norms we needed to prove the local existence theorem do not blow up, and this is sufficient to prove global existence.

Acknowledgement. JB would like to thank the Alexander-von-Humboldt Foundation for the partial financial support he receives as a Feodor Lynen Fellow. 


\section{References}

1. Segal, I.: Ann. Math. 78, 339 (1963)

2. Segal, I.: J. Funct. Anal. 33, 175 (1979)

3. Ginibre, J., Velo, G.: The Cauchy problem for coupled Yang-Mills and scalar fields in the temporal gauge. Commun. Math. Phys. 82, 1 (1981)

4. Eardley, D.M., Moncrief, V.: The global existence of Yang-Mills-Higgs fields in 4-dimensional Minkowski space. I. Local existence and smoothness proporties. II. Completion of proof. Commun. Math. Phys. 83 (1982) 171 and 193

5. Manton, N.S.: A remark on the scattering of BPS monopoles. Phys. Lett. 110B, 54 (1982)

6. Atiyah, M.F., Hitchin, N.J.: Low energy scattering of non-abelian monopoles. Phys. Lett. $107 \mathrm{~A}, 21(1985)$

Communicated by L. Nirenberg

Received October 29, 1985 